\title{
EMPODERAMIENTO DE LA MUJER EN EL ÁREA LABORAL EN MÉXICO
}

\author{
Est. Candra Lizbeth Moreno Rueda ${ }^{1}$ \\ Dr. José Antonio Morales Notario ${ }^{2}$ \\ Dra. Josefina De La Cruz Izquierdo ${ }^{3}$
}

Resumen: El interés de las instituciones internacionales por la equidad entre mujer y hombre es abundante y juega un papel muy importante en el surgimiento del término empoderamiento y equidad en la historia y hasta su actualidad. En el ámbito laboral, el empoderamiento de la mujer surge con la finalidad de lograr un mejor progreso, cambio e igualdad en ellas. La mujer estaba sujeta a muchos factores de afectación personal y es ese empoderamiento, el que le da la oportunidad de abrirse camino para alcanzar la igualdad de género en su crecimiento. Sin embargo, a pesar de ello, el género femenino tiene un papel difícil por diversos factores que impiden su integración de una forma amplia y sana.
Usando el método bibliográfico y deductivo-inductivo, al existir información al respecto, en el presente artículo se analizan las normas nacionales para un enfoque más concreto y de protección para el desarrollo laboral de mujer de forma íntegra; así como factores internacionales que ayudaron a abrir paso a la igualdad laboral y la protección de los derechos de la mujer.

Palabras Clave: Empoderamiento, Igualdad de género, empleo, mujeres.

\begin{abstract}
The interest of international institutions in the equality between women and men is plenty and it plays a very important role in the rise of the term
\end{abstract}

\footnotetext{
${ }^{1}$ Actualmente cursa el Séptimo ciclo de la Licenciatura en Derecho en la División Académica de Ciencias Sociales y Humanidades de la Universidad Juárez Autónoma de Tabasco en México. Colabora en el proyecto: "La igualdad de Género en México" en el Verano de Investigación Científica 2017, del cual deriva la presente investigación.

${ }^{2}$ Doctor en Derecho con Maestría en Administración de Justicia. Es profesor de Tiempo Completo en la División Académica de Ciencias Sociales y Humanidades de la Universidad Juárez Autónoma de Tabasco. Colabora con el Verano de Investigación Científica 2017, de donde deriva el presente artículo.

${ }^{3}$ Doctora en Educación con Maestría en Tecnología Educativa. Es profesora en la División Académica de Ciencias Sociales y Humanidades de la Universidad Juárez Autónoma de Tabasco. Actualmente colabora con el Verano de Investigación Científica 2017, de donde deriva el presente artículo.
} 
empowerment and equity in the history and until its present day. In the work sphere, the empowerment of women appears with the purpose of achieving better progress, change and equality in them. Women were abiding to many factors of personal concern and it is this empowerment, which gives them the opportunity to achieve the gender equality. However, despite this, the female gender has a difficult role due to different factors that prevent its integration in an open and good way. Using a bibliographic and deductive-inductive method, taking in consideration that there is a lot of information about the topic, this article analyzes the national rules for a more concrete and protective approach to the development of women's work in an integral way; as well as international factors that helped to find a way to labor equality and the protection of women's rights.

Keywords: empowerment, gender equality, job, women

\section{INTRODUCCIÓN}

El termino empoderamiento surgió ligado al de igualdad de género, ambos con el objetivo de proporcionar más beneficios a la mujer, para su incorporación de forma equitativa $\mathrm{e}$ igualitaria al área laboral. El surgimiento de ambos conceptos es gracias a las labores de gran impacto de los movimientos feministas en la sociedad y en el mundo; que buscaban permitir a la mujer tener un mejor crecimiento personal, para mejorar su calidad de vida y que pretendían emanciparse del yugo masculino que era el que dictaba las actividades que debían realizar.

Gracias a los intereses de los movimientos nacionales e internacionales, a través de los tiempos surgieron convenios, recomendaciones y más beneficios en cuanto a derechos para la mujer, igualándola con el hombre y permitiéndole tener más acceso al campo del trabajo para su mejor desarrollo.

Desde la Cuarta Conferencia Mundial sobre la Mujer, celebrada en Beijing en 1995 (ODM, s.f.), sólo se ha logrado avanzar en poca medida en los factores que inciden en el ámbito laboral. En gran parte del mundo y en México, la desigualdad entre mujeres y hombres es 
palpable en las oportunidades, el trato y los resultados que ambos esperan obtener y que por cuestiones ajenas no resulta ser el adecuado para el mal llamado género femenino.

En este artículo se analizan los factores que impiden que la mujer pueda ingresar a un trabajo con los mismos beneficios que el hombre y que afectan de manera permanente o temporal su desempeño e impiden un mejor desarrollo laboral; fenómenos de gran impacto que tratan de prevenirse a través de criterios normativos y considerados como parte de los derechos fundamentales del hombre; sin embargo, los mismos han resultado insuficientes para poder lograr un verdadero equilibrio entre los géneros, en los que no haya desigualdad.

La mujer siempre se ha encontrado con la dificultad de poder encontrar un empleo que le satisfaga, (aún más que el hombre), y que le ayude a obtener los beneficios que ella misma busca para ver un cambio en cualquier momento de su vida, como son: un mejor ingreso, las mismas oportunidades de superarse en el ámbito laboral, lograr la igualdad, un mejor trato y más oportunidades, permitiendo reflejar el empoderamiento en ellas; entre muchos más aspectos.

\section{DEFINICIÓN EMPODERAMIENTO}

DE

En el pasado, para que surgiera un concepto de “empoderamiento", se realizó una profunda investigación de la condición en que se encontraban las mujeres, en la que se analizó su situación de pobreza, bienestar familiar, estudios, oportunidades, entre otros aspectos. Este concepto se fortaleció gracias a las labores feministas, interesadas en el género, y en búsqueda de un estado equitativo y de no extorsión. El empoderamiento se manifestó gracias a los cambios que las mujeres crearon para sí mismas en la sociedad a través de diferentes luchas en las que lograron el reconocimiento de sus derechos.

Dentro de las distintas conceptualizaciones que se le da al fenómeno del empoderamiento, Sharma, entre 1991-1992, lo establece como un proceso orientado a cambiar la naturaleza y la dirección de las fuerzas sistémicas, que marginan a la mujer y a otros sectores en desventajas en un contexto dado. El 


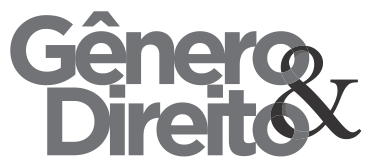

empoderamiento busca cambiar el estado

de marginación en que muchas mujeres se encuentran.

Por otra parte, Murguialday (2006), considera que el empoderamiento de las mujeres contribuye al logro de las metas globales del Desarrollo; y esto es debido a que la propia mujer busca su progreso en la sociedad. Wieringa (1997), indica que el empoderamiento sólo tiene significado cuando se utiliza en el contexto de la transformación social según la concepción feminista del mundo; Young (1997) señala que es una alteración radical de los procesos y estructuras que reproducen la posición subordinada de las mujeres como género.

Por otra parte, la conceptualización de empoderamiento surge desde otras perspectivas, incidiendo en el querer del cambio $y$ de la transformación de la mujer para sobresalir en diferentes ramas de la sociedad como es el área laboral y la política o el hecho de querer un mejor cambio de vida.

Cosude (2004) indica que el empoderamiento es un proceso que contribuye a que las personas y sus organizaciones puedan ser, hacer y decidir por sí mismas; mientras que Ferguson
Cico do Núcleo de Estudos e Pesquisas sobre Gênero e Direito Centro de Ciências Jurídicas - Universidade Federal da Paraíba V. 6 - No 03 - Ano 2017 (Spanish Edition) político y material que aumenta el poder del individuo y del grupo, su resistencia y su capacidad de actuar por sí mismos. Por su parte Oakley (2001) plantea que el empoderamiento sólo logrará cambios significativos si se orienta a transformar las relaciones de poder existentes. En otras palabas, el empoderamiento puede definirse como la expansión en la libertad de escoger y actuar (Banco Mundial, S.F).

Las Naciones Unidas, (DAES, 2005) lo define como los procesos por los cuales las mujeres y los hombres ejercen el control y se hacen cargo de su propia vida mediante la ampliación de sus opciones. Lo predominante es la manifestación de las diferentes conceptualizaciones que puedan denominarse del empoderamiento.

Kabeer (1999), dice que sólo un tanto ha podido retener el dominio sobre su definición, pero estas conceptualizaciones son en conjunto necesarias para el estudio y entendimiento del empoderamiento, porque en cada una de ellas se analiza un aspecto diferente pero necesario para llegar a una sola concretización del tema. Se puede decir por tanto que el empoderamiento es una variable de "poder", porque el termino trata 
primordialmente de eso, de tomar la decisión de que se quiere hacer para mejorar, de tomar el control de la vida propia para su fortalecimiento $\mathrm{y}$ mejoramiento físico, psicológico, pero también, cultural, económico, material, laboral, político, etc.

\section{DEFINICIÓN DE IGUALDAD DE GÉNERO}

La igualdad de género es un término de mucha importancia en materia de Derechos Humanos. En la historia de la humanidad ha prevalecido para el sustento de dar a cada quien lo que le corresponde de forma igualitaria y equitativa. La igualdad de género en sus diferentes conceptualizaciones se instruye a la sociedad en relación a la dignidad humana, manifestada en los últimos cincuenta años por los gobiernos y las sociedades civiles, para evitar las diferencias que a través de los tiempos han surgido entre los géneros.

Por consiguiente, corresponde definir este término para la comprensión del contenido de este artículo;

"Por "género" se entienden
las construcciones
socioculturales que
diferencian y configuran los

roles, las percepciones y los estatus de las mujeres y de los hombres en una sociedad. Por "igualdad de género" se entiende la existencia de una igualdad de oportunidades y de derechos entre las mujeres $y$ los hombres en las esferas privada y pública que les brinde y garantice la posibilidad de realizar la vida que deseen. Actualmente, se reconoce a nivel internacional que la igualdad de género es una pieza clave del desarrollo sostenible". (Secretario General de las Naciones Unidas sobre la sostenibilidad mundial, 2012).

La igualdad de género al igual que muchas conceptualizaciones, es abundante, pero son pocas las que describen lo que realmente es:

"Igualdad de género se define como "la igualdad de derechos, responsabilidades y oportunidades de las mujeres y los hombres, y las niñas y los niños". La igualdad no significa que las mujeres y los hombres sean lo mismo, sino que los derechos, las responsabilidades y las oportunidades no dependen del sexo con el que nacieron. La igualdad de género supone que se tengan en cuenta los intereses, las necesidades y las prioridades tanto de las mujeres como de los hombres, 
reconociéndose la diversidad de los diferentes grupos de mujeres $y$ de hombres" (OSAGI) (UNESCO S.f.).

Por otra parte, la Ley General para la Igualdad de Género entre Mujer y Hombre (2013) en su artículo 5, define la Igualdad de Género como;

"Situación en la cual mujeres y hombres acceden con las mismas posibilidades $y$ oportunidades al uso, control y beneficio de bienes, servicios y recursos de la sociedad, así como a la toma de decisiones en todos los ámbitos de la vida social, económica, política, cultural y familiar".

Por las conceptualizaciones de diferentes autores, la igualdad de género implica los derechos, los deberes y responsabilidades que toda persona tiene en sociedad, y de dar a cada quien lo que le corresponde de una forma equilibrada con el objeto de lograr el bien común que todo Estado busca para vivir en armonía y conforme a derecho.

\section{RELACIÓN ENTRE EL EMPODERAMIENTO DE LA MUJER Y LA IGUALDAD DE GÉNERO}

En este tema, una figura importante es el Objetivo de Desarrollo del Milenio (ODM), (ONU s.f.), el cual es una declaración donde participan los países integrados a la ONU, entre ellos México. El tercer objetivo llama a la promoción de la igualdad de género y el empoderamiento de la mujer, ya que es necesario lograrlos para alcanzar las metas del desarrollo humano. Por otra parte, la igualdad es un derecho humano fundamental. Promoverla es esencial en todos los ámbitos de una sociedad sana: desde la reducción de la pobreza hasta la promoción de la salud, la educación, la protección y el bienestar de todos. En ese mismo sentido, el empoderamiento y la igualdad de género buscan un cambio y un bienestar.

Tanto la igualdad de género como el empoderamiento trabajan de la mano para un cambio oportuno que se viene dando desde épocas históricas en manifestaciones y convenciones; y son la base necesaria para conseguir un mundo, próspero y sostenible, así como el facilitar a las mujeres y niñas la igualdad en el acceso a la educación, atención médica, un trabajo decente y representación en los procesos de adopción de decisiones políticas y económicas, (ONU, ODM, s.f.), 
en donde se beneficiará a las sociedades y

a la humanidad en su conjunto; esto sin descuidar al género masculino. Lo que trata de lograr la igualdad entre hombres y mujeres es un equilibrio entre estos dos, con la finalidad de erradicar las condiciones inferiores de uno sobre el otro que en muchas ocasiones los coloca en una situación desventajosa de uno con respecto al otro.

\section{LA MUJER EN EL ÁMBITO}

\section{LABORAL}

En México hay un alto índice de mujeres que trabajan para obtener un mejor sustento para sus familias.

"Durante las últimas tres
décadas, [...] México ha
registrado un incremento
sostenido en la tasa de
participación de las mujeres
en el empleo: en 1970, 17 de
cada 100 mujeres
desarrollaban actividades
económicas, pero en la
actualidad el número se ha
incrementado a 35, (Inmujeres
2013).

El índice de empoderamiento de la mujer (de querer el cambio para un mejor modo de vida) crece a medida de la necesidad salarial, de obtener un ingreso para su sustento, y el de sus familias. Todo por las trasformaciones económicas que el país ha tenido dentro del contexto de la globalización y como se observa en esta época, la crisis salarial del país obliga el crecimiento de la figura femenina en las áreas laborales.

Los estudios de la integración de las mujeres en el mercado de trabajo en México demuestran la existencia de dos modalidades de segregación o separación ocupacional por géneros: una, que atañe a la concentración de la fuerza de trabajo femenina en unas cuantas ocupaciones y profesiones, y otra, que corresponde al lugar o la ubicación de las mujeres en las jerarquías establecidas, en los distintos centros de trabajo.

Los ingresos que obtienen las mujeres, no permiten una apreciación optimista de los cambios que cada mujer se propone obtener cuando decide ingresar a trabajar. Los factores a los que toda mujer se puede ver sujeta al momento de insertarse en un empleo pueden ser los siguientes:

\section{DESIGUALDAD LABORAL}




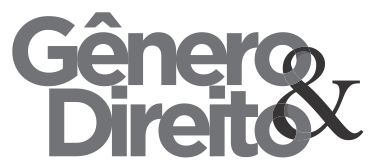

A lo largo de su trayectoria laboral, las mujeres siguen experimentando grandes dificultades para ingresar a empleos en los que se reconozca su valor intelectual; en ello inciden diversos factores que impiden que desarrolle una actividad en este ámbito; por ello, el empoderamiento ha sido un mecanismo que les ha permitido imponerse ante aquellos hombres que las discriminan o no valoran sus conocimientos y con el que buscan combatir el conflicto generado entre géneros, con lo que buscan mejorar y realizar una actividad laboral en la que puedan desarrollarse y lograr un verdadero cambio de condiciones.

Los principales factores que inciden son; el acoso laboral, diferencias de sueldos, grado académico, la maternidad, la salud, la edad, entre otros.

El acoso laboral podría colocarse como uno de los factores primordiales de impedimento para que una mujer trabaje ya que siempre se encuentra sujeta a este riesgo, en donde muchas veces por la necesidad se ven obligados a permitir este tipo de abuso. Este consiste en el comportamiento hostil, vejatorio o agresivo por parte de un superior o
Codico do Núcleo de Estudos e Pesquisas sobre Gênero e Direito Centro de Ciências Jurídicas - Universidade Federal da Paraíba V. 6 - No 03 - Ano 2017 (Spanish Edition)

personal del mismo nivel jerárquico, hacia un trabajador, generalmente mujer, que causa problemas psicológicos y profesionales y que inciden en su desempeño laboral, familiar, social, cultural y económico, ante la amenaza de represión en caso de que no ceda a las exigencias del acosador.

El grado académico, en la actualidad, es motivo de muchas discusiones, pues la educación se considera indispensable para un mejor $\mathrm{y}$ adecuado ingreso económico, en base a la obtención de un grado académico. Sin embargo, este es un factor que incide en la contratación de las mujeres porque por un lado, no todas tienen la oportunidad de estudiar y las que estudian, tienen que luchar contra los estigmas o estereotipos que la propia sociedad ha creado. Cuando se observa a una mujer en un puesto importante, no se valora su conocimiento sino las relaciones que tiene para llegar a ese lugar o bien, las acciones que tuvo que realizar, como lo es, la posibilidad de que se haya "acostado" con el jefe o alguna otra persona, que le haya ayudado a escalar la posición en la que se encuentra.

La diferencia de sueldos es un factor que desanima e impide que la mujer 
ingrese a un trabajo. En la actualidad, ellas pueden ganar hasta un $17.5 \%$ menos que el hombre, en mandos medios $\mathrm{y}$ altos (CNDH, 2017). Lo anterior debido a que existe todavía la creencia de que los ingresos de la mujer deben ser menores pues sus necesidades con respecto al hombre son diferentes, además de que se menosprecia, como se señaló anteriormente, su capacidad intelectual para desarrollar alguna actividad que el otro género conoce o desarrolla.

La protección de la maternidad tiene como prioridad evitar que la mujer sufra un trato desigual en el empleo por su función natural reproductiva; la ley otorga ciertas consideraciones hacia la futura madre con la finalidad de lograr que el producto sea viable; sin embargo, esto no significa que no sea capaz de desarrollar su trabajo de manera adecuada. En ese sentido, muchos varones ven en esta condición femenina un motivo de desigualdad, lo que es erróneo pues no se consideran cuestiones de género sino reproductivas; por ello, se ha considerado que:

"La maternidad es un estado que exige un trato diferente para poder respetar una auténtica igualdad y, en tal sentido, es más una premisa del principio de igualdad que una excepción del mismo. Se han de tomar medidas especiales de protección de la maternidad para que las mujeres puedan cumplir su función de madres, sin que resulten marginalizadas del mercado de trabajo". (Oficina Internacional del Trabajo, 1996).

Desafortunadamente esto es un factor de discriminación porque incide en la terminación y en el ingreso a un empleo, y si el embarazo se da en el transcurso del desarrollo de un empleo, éste la lleva a pedir su renuncia o es causa de un despido injustificado.

En toda área laboral deben adoptarse medidas para que la "maternidad no constituya una causa de discriminación", que permita a la mujer integrarse a él, eliminando la idea de que ser madre es un impedimento para el desarrollo de su trabajo de forma normal y eficaz.

La salud es un estado físico y/o psicológico que permite a la persona disfrutar plenamente de su fortaleza para un buen desarrollo en el futuro, sin sentirse agobiados por una afectación en su 
persona que lo incapacite temporal o permanentemente.

La salud del trabajador influye de forma inesperada, debido al estereotipo de que, quienes tienen problemas de salud deben tener más derecho a ingresar a un trabajo; aunque en la realidad laboral, es todo lo contrario, ya que el estado de salud influye en el trabador o trabajadora para el desempeño de las actividades asignadas de forma eficaz, ya que una mala salud puede afectar su desempeño físico-psicológico de forma adecuada.

La edad es otro factor de discriminación, sobre todo en los jóvenes y en aquellos de edad avanzada. En el caso de la mujer, se agrega otro elemento: el simple hecho de ser mujer. Desde ese punto de vista, la edad tiene dos variantes: primero, en cuanto a los menores, surge a partir de la protección de los derechos del niño, donde la ley claramente impide que el menor trabaje, a menos que su tutor lo autorice o que dicha cuestión laboral sea bajo su vigilancia y autorización. En la segunda variante se encuentra la vejez, considerando que las empresas establecen que el esfuerzo y rendimiento en el trabajo disminuye y se dificulta llevar a cabo las tareas asignadas por su patrón, impidiendo esto el ingreso a un empleo. En el caso de las mujeres, este factor incide aún más ya que en la sociedad siguen siendo vistas como el sexo débil y combinándolo con la vejez, se dificulta conseguir un empleo bien remunerado. El área laboral exige el rendimiento y la producción del trabajador en gran cantidad. Por ello, la edad constituye un rasgo más de discriminación, que a criterio de muchas personas se acepta como razonable, debido a la capacidad de la mujer para llevar a cabo una actividad de mejor desempeño, y esto en nuestra legislación aún no se "defiende" como otros movimientos hacia la discriminación, los cuales se mencionaron anteriormente.

Otra causa de afectación hacia la mujer, para ingresar a un trabajo o para su realización una vez de haber ingresado a él, son las actividades o responsabilidades en el hogar. El reparto desigual de las labores de cuidado y las tareas domésticas no remuneradas entre las mujeres y los hombres, y entre las familias y la sociedad, son principalmente un contexto de las desigualdades de género que afectan en el trabajo.

La mujer se sujeta a diversos cambios e impedimentos que pueden 
poner obstáculos en la realización de su desempeño laboral. La igualdad de género ha venido defendiendo a través de los tiempos, los mismos derechos que la mujer $\mathrm{y}$ el hombre tienen para lograr una sociedad equitativa. Por medio de las manifestaciones feministas del país y del mundo se han logrado defender dichos derechos, erradicar paso a paso la desigualdad laboral e imponer el empoderamiento para obtener las mismas oportunidades que el hombre.

\section{ORGANIZACIÓN INTERNACIONAL DEL TRABAJO (O.I.T.)}

La Organización Internacional del Trabajo, es un organismo de las Naciones Unidas, especializado y capacitado para ocuparse de los asuntos referentes al trabajo y a las relaciones laborales. Fue fundada en 1919, en virtud del Tratado de Versalles que señalaba que “después de una guerra destructiva, basada en una visión según la cual una paz duradera y universal sólo puede ser alcanzada cuando está fundamentada en el trato decente de los trabajadores" (OIT, 2017).
La OIT ha formado parte fundamental en la promoción del respeto y la igualdad de trato en el área laboral entre el hombre y la mujer, por medio de convenios y recomendaciones que ha emitido desde el surgimiento de este fenómeno.

En la Conferencia General de la OIT, (1991) se hizo la recomendación número 90; (Recomendación sobre la igualdad de remuneración entre la mano de obra masculina y la mano de obra femenina por un trabajo de igual valor). Que a como menciona Castro (2001), se refiere expresamente a la igualdad de trato en materia de empleo y ocupación.

En 1981 se dio la recomendación número 165, sobre los trabajadores con responsabilidades familiares.

En 1980, la OIT emitió la recomendación número 162, que versa sobre los trabajadores de edad avanzada, la cual se aplica a todos los trabajadores que, por su edad, están expuestos a encontrar dificultades en materia de empleo y ocupación.

Con respecto al género, la OIT (2015) argumenta;

"La importancia que revisten la igualdad de género y su contribución al logro de los 17 
Objetivos de Desarrollo Sostenible. La creación de más y mejores empleos para las mujeres, la protección social universal, y la adopción de medidas para reconocer, reducir y redistribuir las labores de cuidado y las tareas domésticas no remuneradas son indispensables para hacer realidad la nueva $y$ transformadora agenda para el desarrollo sostenible, que tiene por objeto reducir la pobreza (Objetivo 1) y las desigualdades (Objetivo 10), lograr la igualdad de género (Objetivo 5), y promover el crecimiento económico sostenido, inclusivo $y$ sostenible, el empleo pleno y productivo y el trabajo decente para todos (Objetivo 8)".

El objetivo de la Organización Internacional del Trabajo es apoyar al trabajador, permitiendo más beneficios a la mujer para hacer valer sus derechos y evitar el abuso, ya que el género femenino está más expuesta a sufrir situaciones de este tipo, (el que no se le permita que haga valer sus derechos y se le abuse). La OIT permite a su vez, que las mujeres se empoderen para lograr su independencia laboral, mejorar su situación; y lograr la igualdad de género en el trabajo.

\section{LAS}

\section{CONVENCIONES}

\section{INTERNACIONALES}

Existen innumerables convenciones realizadas en el mundo con el objeto de beneficiar, proteger y ayudar a la mujer para una mejor aceptación en el trabajo que le permita un buen desarrollo laboral.

Las convenciones internacionales son los instrumentos negociados a través de un patrocinio por medio de una organización o de los propios Estados, con la finalidad de beneficiar a la sociedad en general. En cuanto al género, las Convenciones que buscan promover la igualdad de género y el empoderamiento de la mujer en el área laboral son:

a) La Convención Interamericana Para Prevenir, Sancionar, y Erradicar la Violencia contra la Mujer, conocida como "Convención De Belem Do Pará"; aprobada el 9 de junio de 1994 por la Asamblea General de Estados Americanos (OEA), en su vigésimo cuarto período de sesiones, representa un valioso instrumento jurídico para las mujeres, pues establece a nivel mundial, los parámetros 
legales en torno a la violencia contra la mujer y al cual quedan sujetos todos los países signatarios de dicha Convención.

b) La Conferencia Internacional de Población y Desarrollo (CIPD), realizada en El Cairo en 1994, representa también un avance más a nivel mundial, en el reconocimiento de los derechos humanos de las mujeres, al plasmarse un Programa de Acción que establece especialmente, los derechos de las mujeres en el área desigualdad y equidad; en el acceso a la toma de decisiones; en los derechos de salud sexual y derechos reproductivos y en el área de la violencia contra la mujer.

c) La Declaración Universal de Derechos Humanos, aprobada por la Asamblea General de la ONU, en su Resolución 217 del 10 de diciembre 1948, constituye el documento jurídico base, sobre el que se trabaja el tema de los derechos humanos a nivel legislativo.

El interés que hay en el ámbito internacional por mantener la igualdad a beneficio de la mujer se ve reflejada en: la Plataforma de Acción Mundial de Beijing; la Plataforma de Acción de la Conferencia Mundial de Población del Cairo y la Convención contra la eliminación de todas las formas de Discriminación Contra las Mujeres.

Las diversas Convenciones Internacionales de la mujer, manifestadas a través de la historia, se realizaron con el propósito de crear la igualdad de derechos entre el hombre y la mujer, y en la actualidad se deben reforzar para erradicar las desigualdades que siguen establecidas en el área laboral y la nuevas desigualdades que van surgiendo conforme a las situaciones actuales de la sociedad

El tema de las convenciones internacionales es muy amplio y a la vez muy importante en relación al empoderamiento de la mujer y la igualdad en el trabajo, ya que hay que tener siempre en cuenta que la igualdad y el empoderamiento son temas que van de la par en contexto nacional e internacional; que buscan fortalecer los derechos entre los hombres y las mujeres, para que ella logre un medio de vivir digno, estable, con una remuneración justa y dónde se erradique la desigualdad y se logre la equidad.

\section{LAS LEYES NACIONALES}




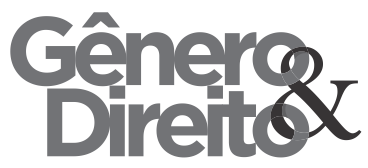

En México hay leyes que

protegen los derechos de las personas en el área laboral y en el ámbito de la igualdad. La Constitución Política de los Estados Unidos Mexicanos al igual que los Tratados Internacionales se encuentra en un mismo rango de jerarquía.

La Constitución es la carta suprema y la más importante en proteger los derechos de los ciudadanos. En su artículo 123 se protegen los derechos del trabajador. En su artículo 4, se privilegia la igualdad de género y de ella emanan las demás leyes locales.

Desde un punto de vista personal, el empoderamiento de la mujer para las manifestaciones de igualdad en México se da a partir del artículo primero constitucional cuando prohíbe todo tipo de discriminación motivada por origen étnico o nacional, por el género, la edad, las discapacidades, la condición social, las condiciones de salud, la religión, las opiniones, las preferencias sexuales, el estado civil o cualquier otra que atente contra la dignidad humana y tenga por objeto anular o menoscabar los derechos y libertades de las personas. (Const., 2017, art. 1).
Cen do Núcleo de Estudos e Pesquisas sobre Gênero e Direito Centro de Ciências Jurídicas - Universidade Federal da Paraíba V. 6 - No 03 - Ano 2017 (Spanish Edition)

El artículo 123 del instrumento jurídico en análisis le da los mismos derechos a la mujer y al hombre en la forma de trabajar, siempre que ésta sea de manera digna y útil. El apartado A fracción $\mathrm{V}$ establece los derechos de la madre trabajadora que se encuentra en estado de gestación y en periodo de lactancia.

La Ley Federal del Trabajo, creada para proteger los derechos de todo trabajador así como sus obligaciones, en su artículo 56 establece la igualdad plena de los sexos en relación al trabajo. El Título Quinto hace referencia al trabajo de la mujer con relación a lo estipulado en el artículo 123 de la Constitución.

Ahora bien, en México las jurisprudencias emitidas por la Suprema Corte de Justicia de la Nación, que son criterios orientadores para resolución de casos y que son obligatorias una vez publicados en el Semanario Judicial de la Federación, han establecido parámetros para la igualdad de género laboral:

"Derecho humano a la igualdad entre el varón y la mujer. $\mathrm{Su}$ alcance conforme a lo previsto en el artículo $4 o$. de la constitución política de los estados unidos mexicanos y en los tratados internacionales". 


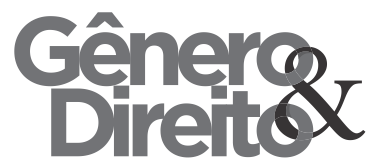

(Corte Suprema de Justicia, Primera Sala Constitucional, 2014099, 2017).

Esto es derivado del artículo 4 constitucional que establece que el hombre y la mujer son iguales ante la ley.

Las leyes nacionales, al igual que las internacionales, protegen los derechos de igualdad del hombre y la mujer en el contexto laboral, todo para obtener un estado solidario $y$ sin diferencia $y$ discriminación. Si una mujer sufre de algún abuso o discriminación puede acudir a instituciones como la Comisión Nacional de los Derechos Humanos (CNDH), para proteger sus derechos conforme a lo estipulado en la constitución.

\section{PROPUESTAS Y CONCLUSIONES}

Las propuestas en base al tema principal, empoderamiento de la Mujer en el Área Laboral son;

1. Es necesario que las mujeres se informen acerca de los principios para el empoderamiento que piden las empresas y conozcan los requisitos que solicitan las área de reclutamiento para ingresar a un trabajo; así tendrán la facilidad de
Cedico do Núcleo de Estudos e Pesquisas sobre Gênero e Direito Centro de Ciências Jurídicas - Universidade Federal da Paraíba V. 6 - No 03 - Ano 2017 (Spanish Edition) acceder y ascender más rápido en el área laboral que escojan o le designen para así obtener un mejor ingreso económico y lograr lo que el empoderamiento pretende;

2. Que las mujeres estén más preparada académicamente para que se amplíen las oportunidades de ingresar al área laboral;

3. Que se siga promoviendo la igualdad de género mediante instituciones y convenciones para que el hombre y la mujer tengan los mismo beneficios en sociedad;

4. Que conforme a las leyes, la Igualdad salarial sea respetada, pero sobre todo ejercida $\mathrm{y}$ protegida ante la ley.

Como resultado de la investigación, es posible concluir que existe una relación entre el empoderamiento de la mujer en el área laboral y la igualdad de género. Una no puede surgir sin la otra, por lo que existe una codependencia; pero tampoco podemos negar que sigue existiendo la desigualdad a pesar de las normas y las leyes. 


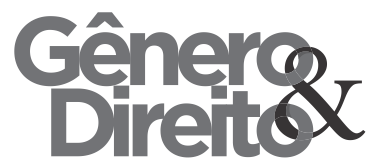

En muchos países, la desigualdad

en la participación de la fuerza de trabajo y el desempleo se ha reducido gracias a la figura de empoderar, y las mujeres están dejando de lado el trabajo familiar para centrarse más en el sector de los servicios, pero la calidad de los empleos de las mujeres sigue suscitando preocupación.

Los notables progresos en movimientos por mujeres para hacer que sus derechos se hagan valer y se fortalezcan la igualdad, hoy ha rendido frutos y además, están dando resultados.

La sociedad nacional e internacional tiene mucho que hacer para que la igualdad de género se siga promoviendo y que junto con el empoderamiento siga creando cambios para el bien común. Tan pronto se vayan creando las condiciones adecuadas para lograr el empoderamiento de la mujer de manera positiva, mayor será el progreso en materia de protección y defensa de los Derechos Humanos.

\section{REFERENCIAS BIBLIOGRÁFICAS}

Argüello, N. S. (2011). La edad como factor de discriminación en el empleo.
Cédico do Núcleo de Estudos e Pesquisas sobre Gênero e Direito Centro de Ciências Jurídicas - Universidade Federal da Paraíba V. 6 - No 03 - Ano 2017 (Spanish Edition)

Revista Universitaria de Ciencias del

Trabajo, 8.

Batliwala, S. (1997).

http://www.congresoed.org. Obtenido de http://www.congresoed.org:

http://www.congresoed.org/wp-

content/uploads/2014/10/D4_Batliwala_1

997.pdf

Cámara de Diputados del H. Congreso de la Unión, Diario Oficial de la Federación, (2 de agosto de 2006) Ley General para la Igualdad entre Mujeres y Hombres. (Ley

5 de 2006) DOF 14-11-2013.

Cámara de Diputados del H. Congreso de la Unión, Diario Oficial de la Federación, (2017) Ley Federal del Trabajo. (Ley 56 de 2017) DOF 2017.

Castro, J. F. (marzo de 2001). http://www.dt.gob.cl. Obtenido de http://www.dt.gob.cl/1601/articles$\underline{65173 \text { recurso_1.pdf }}$

Constitución Política de los Estados

Unidos Mexicanos [Const.] (2017)

Artículo 1, [Título I]. Ultima Ed. Legis. 


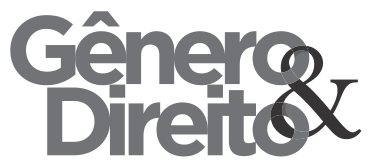

Corte Suprema de Justicia, Primera Sala

Constitucional, (abril-201), 2014099,

(Jurisprudencia).

De Rham Philipp Crespo Patricio, I. P.

(octubre de 2007).

http://www.asocam.org. Obtenido de

http://www.asocam.org:

http://www.asocam.org/biblioteca/ASO_

RA_Empoderamiento.pdf

IInmujeres. (2003). Las mexicanas y el

trabajo www.inmujeres.gob.mx

http://cedoc.inmujeres.gob.mx/documento

s_download/100500.pdf

Igualdad de Género, indicadores unesco

de cultura para el desarrollo, (s.f.).

http://es.unesco.org/creativity/sites/creati

vity/files/digitallibrary/cdis/iguldad\%20d

e\%20genero.pdf

Martínez, C. M. (2006).

http://www.vitoria-gasteiz.org.

Recuperado el 22 de junio de 2017, de

http://www.vitoria-gasteiz.org:

http://www.vitoria-

gasteiz.org/wb021/http/contenidosEstatic

os/adjuntos/es/16/23/51623.pdf
Cónco do Núcleo de Estudos e Pesquisas sobre Gênero e Direito Centro de Ciências Jurídicas - Universidade Federal da Paraíba V. 6 - No 03 - Ano 2017 (Spanish Edition)

ONU (s.f.). Los objetivos de desarrollo del milenio en américa latina y el caribe;

promover la igualdad de género y el empoderamiento de la mujer

http://www.bvsde.paho.org/bvsacd/mileni

o/cap6.pdf

Organización I, T. (2016). Resumen

ejecutivo.

http://www.ilo.org/wcmsp5/groups/public /dgreports/dcomm/publ/documents/public ation/wcms_457094.pdf

O.I.T. Ginebra, Suiza. (1985). Convenios

y recomendaciones internacionales del trabajo.

http://www.ilo.org/dyn/normlex/es/f?p=N

ORMLEXPUB:12100:0::NO::P12100_IN

STRUMENT_ID:312428

OIT. (2010). http://www.ilo.org. Obtenido de

http://www.ilo.org/wcmsp5/groups/public /---dgreports/---dcomm/--publ/documents/publication/wcms_14215 9.pdf

Piras, C, y Vega, G. (s.f.). Los objetivos de desarrollo del milenio en américa latina y el caribe. 

V. 6 - No 03 - Ano 2017 (Spanish Edition)

ISSN | 2179-7137 | http://periodicos.ufpb.br/ojs2/index.php/ged/index

http://www.bvsde.paho.org/bvsacd/mileni

o/cap6.pdf

Unidas, S. G. (2012). http://www.un.org.

Obtenido de http://www.un.org:

http://www.un.org/gsp/sites/default/files/a

ttachments/Overview\%20\%20Spanish.pd

f. 